\title{
THE SCALING RELATIONS FOR CLUSTERS OF GALAXIES
}

Fundamental Planes, Cluster Structure, and Cluster Evolution

\section{J. ANNIS}

Experimental Astrophysics Group, Fermilab

\section{Introduction}

The dominant baryonic component of clusters of galaxies is their X-ray emitting atmosphere. X-ray surveys have made it possible to examine this component in large samples of clusters. David et al. (1993) provided a catalog of X-ray temperatures. I have measured 79 clusters from that list using Einstein IPC data (Harris et al. 1990), determining several measures of the radius. The most useful turns out to be the Petrosian radius. Given a radius, one can measure the surface brightness at that position.

\section{Fundamental Planes}

The set of observables $r_{p}-I_{p}-T_{x}$ are interrelated in such a way that given any two I can predict the third. This is at root the result of the virial theorem implying that $r \propto T \eta^{-1}$, and the bremsstrahlung expression for surface brightness, $I_{p} \propto r_{p}^{-1} T_{x}^{1 / 2} \eta^{2}$ (where $\eta$ is the mass surface density). The relationship I measure is

$$
r_{p} \propto T_{x}^{1.30} I^{-0.42}
$$

This relation differs from the virial in a way that can be understood as a variation of $M_{l u m}$ with $M_{t o t}$.

In practice, any mass indicator may be used in place of $T_{x}$, including $L_{x}$ and $N_{A}$. The fundamental plane relationships of $r_{p}-I_{p}-T_{x}$ and $L_{x}-I_{p}-T_{x}$ can be used as distance indicators. The width of both are consistent with the measurement error on $T_{x}$, so they can probably be improved with better measurements of $T_{x}$. 


\section{Cluster Structure}

I have found two exotic distance indicators, relations that seem not to be the result of the virial theorem, but rather related to cluster structure. These are the $r_{p}-I_{m} / I_{p}$ and $L_{x}-I_{m}$ relations. ( $I_{m}$ is the surface brightness at 250 kpc.) The $L_{x}-I_{m}$ relation in particular seems to suggest a quite stunning thing. It can be understood if the dominant contributor to the luminosity in all clusters is distributed with the same functional form and similar scale radii. And indeed, the four very different clusters of Jones and Forman have profiles that, while differing in detail, are roughly straight lines of constant slope.

\section{Cluster Evolution}

We can use distance measuring to look for evolution. The methodology is the same as if I was trying to measure $q_{0}$. The distance indicator relations are linear in log-log plots. I assume evolution occurs in the form $r \propto$ $r_{\star}(1+z)^{a}, L \propto L_{\star}(1+z)^{b}$, and $T \propto T_{\star}(1+z)^{c}$. Evolution then appears as a shift in the zeropoint of the distance indicator relation as a function of $z$.

Each distance indicator has a different sensitivity to evolution. The fundamental plane indicators are primarily sensitive to $T_{x}$ evolution, though there is also sensitivity to $L_{x}$. The "exotic" indicators are primarily sensitive to $r_{p}$ evolution. The data are consistent with no evolution in $T_{x}$, but they are also consistent with evolution in both $L_{x}$ and $T_{x}$ that balance each other. The data are consistent with little or no evolution in $r_{p}$.

The Einstein sample reported here cannot place too strong a constraint on cluster evolution for two reasons: a) there are only a handful of clusters at $z>0.15$, and $\mathrm{b}$ ) the selection criteria for the $z>0.15$ clusters was haphazard at best. A further analysis with ROSAT and ASCA data promises to place stringent limits.

\section{Summary}

Studying clusters in the X-ray reveals a very powerful set of scaling relations. A full report on the study sketched here can be found in Annis 1996.

\section{References}

Annis, J. 1996 Astrophys.J., submitted.

David, L. P., Slyz, A., Jones, C., Forman, W., Vrtilek, S. D. and Arnaud, K. A. 1993 Astrophys.J. 412479

Harris, D. E. et al. 1990, The Einstein Observatory Catalog of IPC X-ray Sources (Smithsonian Institution Astrophysical Observatory) 